\title{
Potential Detection of Secular Evolution of Chemical Abundances on the Surface of the HgMn Primary in the Triple System $\chi$ Lup
}

S. P. Järvinen ${ }^{1}$ (D), S. D. Chojnowski² iD, J. F. González ${ }^{3}$, S. Hubrig ${ }^{1}$ iD, M. Schöller ${ }^{4}$, I. Ilyin ${ }^{1}$, C. A. Hummel ${ }^{4}$ iD, H. Korhonen ${ }^{5}$, and R.-D. Scholz ${ }^{1}$ iD

Published 2020 January 16 • (c) 2020. The American Astronomical Society. All rights reserved. Research Notes of the AAS, Volume 4, Number 1

sjarvinen@aip.de

${ }^{1}$ Leibniz-Institut für Astrophysik Potsdam (AIP), An der Sternwarte 16, D-14482

Potsdam, Germany

2 Apache Point Observatory and New Mexico State University, P.O. Box 59, Sunspot, NM 88340-0059, USA

3 Instituto de Ciencias Astronómicas de la Tierra y el Espacio, Av. España 1512 sur, San Juan, Argentina

${ }^{4}$ European Southern Observatory, Karl-Schwarzschild-Str. 2, D-85748 Garching, Germany

${ }^{5}$ DARK, Niels Bohr Institute, University of Copenhagen, Lyngbyvej 2, DK-2100

Copenhagen, Denmark

S. P. Järvinen iD https://orcid.org/0000-0003-3572-9611

S. D. Chojnowski (iD https://orcid.org/0000-0001-9984-0891

S. Hubrig (iD https://orcid.org/0000-0003-0153-359X

C. A. Hummel (D) https://orcid.org/0000-0002-4308-0763

R.-D. Scholz (iD https://orcid.org/0000-0002-0894-9187

Received 2020 January 13

Accepted 2020 January 15

Published 2020 January 16 
S. P. Järvinen et al 2020 Res. Notes AAS 411

https://doi.org/10.3847/2515-5172/ab6bd2

Spectroscopic binary stars ; Variable stars ; Chemically peculiar stars

\begin{tabular}{lll} 
Export citation and abstract & BibTeX \\
\hline
\end{tabular}

$\chi$ Lup (=HD 141556) is a bright $(V=3$ m95) double-lined spectroscopic binary with an orbital period of 15.26 days (e.g., Dworetsky 1972; Le Bouquin et al. 2013) and a primary belonging to the mercury-manganese (HgMn) peculiarity class (e.g., Wahlgren et al. 1994). HgMn stars are known to exhibit large overabundances of $\mathrm{Hg}$ and $\mathrm{Mn}$ and, similar to magnetic Ap/Bp stars, are spectrum variables, implying the presence of an inhomogeneous element distribution over their surfaces (e.g., Hubrig \& Mathys 1995). A few HgMn binary systems have previously been reported to exhibit dynamical and secular evolution of surface chemical spots and chemical abundances on a timescale of a few months to several years (e.g., Hubrig et al. 2010; Korhonen et al. 2013).

The HgMn primary in the system $\chi$ Lup is of special interest for spectroscopic studies, as it is one of the coolest known HgMn stars and exhibits remarkable abundance anomalies, including isotopic compositions of $\mathrm{Hg}$ and Pt extremely deviating from the terrestrial composition (Hubrig et al. 1999). According to Castelli, the secondary in the system $\chi$ Lup is also chemically peculiar (http://wwwuser.oats.inaf.it/castelli/hd141556/hd141556.html). Three highresolution High Accuracy Radial velocity Planet Searcher (HARPS; Snik et al. 2008) spectra were obtained in 2009, 2010 May, and 2014 August on very similar orbital phases, between 0.71 and 0.74 . The observed distinct changes in line profiles belonging to different elements are presented in the left of Figure 1 and indicate a potential secular evolution of their abundances. Evidently, different elements show abundances that change with time and their line profiles show very different variability behavior. The highest abundances are observed in the spectrum obtained in 2009. The second highest abundances are observed in 2010 for $\mathrm{Hg}$ and Y, but not for Pt (see the Pt II 4047 line). The abundances of iron-peak elements are similar in the 
spectra obtained in 2010 and 2014. We note that future spectroscopic work is necessary to settle the issue of the possible subsynchronous rotation mentioned by Le Bouquin et al. (2013). In case of subsynchronous rotation, the detected variability can also be related to surface spot visibility.

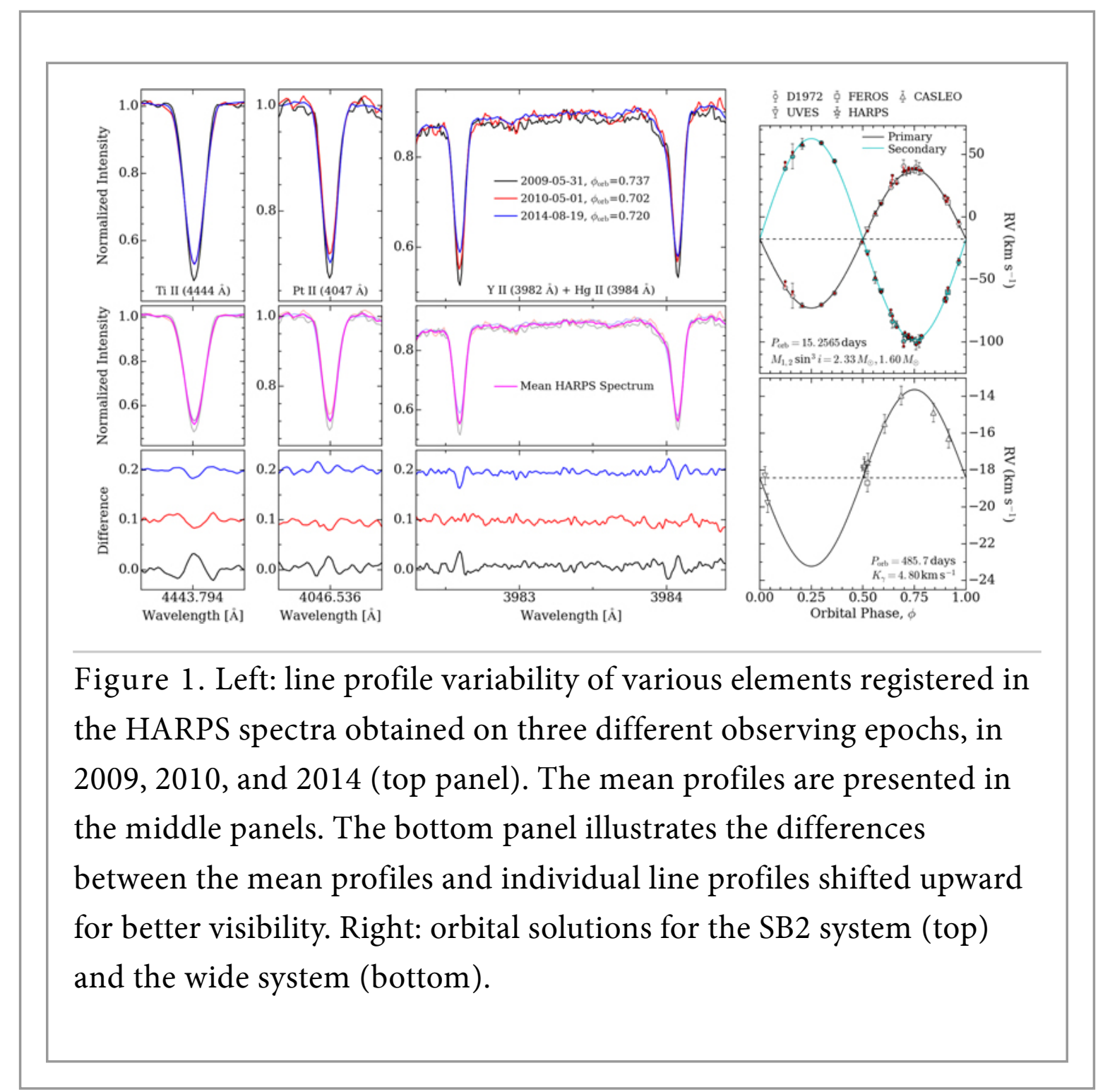

Dworetsky (1972) reported that the $\gamma$ velocity of $\chi$ Lup is variable, indicating the possible presence of a third body, which is invisible spectroscopically. Hubrig et al. (2001) and Schöller et al. (2010) used the ESO adaptive optics instruments ADONIS and NAOS-CONICA, with a spatial resolution down to 54 mas, but could not detect a visual companion to $\chi$ Lup. Also the interferometric observations carried out by Le Bouquin et al. (2013) using PIONEER at the Very Large Telescope did not reveal a third body. The authors reconstructed the three-dimensional orbit of the SB2 system with an orbital 
period of 15.256560 days and estimated a mass of $2.84 M_{\odot}$ for the primary and $1.94 M_{\odot}$ for the secondary. To confirm the presence of the third component, we checked the radial velocities of both components on all spectra available to us. In addition to the HARPS spectra, we downloaded one FEROS spectrum recorded in 2013 February and two UVES spectra obtained in 2006 March on two consecutive nights. Four additional observations of $\chi$ Lup were obtained in 2017 July and 2018 March at a lower resolution of about 13,000 using the CASLEO $2.1 \mathrm{~m}$ telescope.

We converted the pairs of radial velocity measurements into $\gamma$ velocities, using the mass ratio between primary and secondary, as given by Le Bouquin et al. (2013). Fitting the values from the spectra listed above, we determined a period of 485.7 days for the variation of the $\gamma$ velocities. The older data used by Dworetsky (1972) are consistent with this result. For the semi-amplitude K1, we obtain $4.8 \mathrm{~km} \mathrm{~s}^{-1}$, and the mass function for the system $\mathrm{AB}+\mathrm{C}$ is $f\left(m_{\mathrm{A}}+m_{\mathrm{B}} m_{\mathrm{C}}\right)=0.0055 M_{\odot}$, assuming $e=0$. Due to the degeneracy between the inclination of the outer orbit and the mass of the tertiary component, we can give only pairs for these values, e.g., for an orbital inclination of $i=30^{\circ} 7$, the secondary mass is $1.15 M_{\odot}$, and for $i=50^{\circ}$, the secondary mass is $0.73 M_{\odot}$. Spectroscopic orbits of the SB2 system and the wide system are presented in the right of Figure 1.

Based on observations made in CASLEO, operated under agreement between CONICET of Argentina and National Universities of La Plata, Córdoba, and San Juan, and using ESO telescopes under programmes 076.D-0169, 083.D-1000, 085.D-0296, 090.D-0358, and 093.D-0833.

\section{References}

个 Dworetsky M. M. 1972 PASP 84254

IOPscience (https://iopscience.iop.org/1538-3873/84/498

1254) ADS Google Scholar

^ Hubrig S., Castelli F. and Mathys G. 1999 A\&A 341190

ADS Google Scholar

؟ Hubrig S., Le Mignant D., North P. and Krautter J. 2001 A\&A 372152 
Crossref ADS Google Scholar

^ Hubrig S. and Mathys G. 1995 ComAp 18167 ADS Google Scholar

^ Hubrig S., Savanov I., llyin I. et al 2010 MNRAS 408 L61

Crossref ADS Google Scholar

؟ Korhonen H., González J. F., Briquet M. et al 2013 A\&A 553 A27

Crossref ADS Google Scholar

个 Le Bouquin J.-B., Beust H., Duvert G. et al 2013 A\&A 551 A121

Crossref ADS Google Scholar

^ Schöller M., Correia S., Hubrig S. and Ageorges N. 2010 A\&A 522 A85 Crossref ADS Google Scholar

S Snik F., Jeffers S., Keller C. et al 2008 Proc. SPIE 7014 E22 ADS Google Scholar

؟ Wahlgren G. M., Adelman S. J. and Robinson R. D. 1994 ApJ 434349 Crossref ADS Google Scholar

\begin{tabular}{lll} 
Export references: & BibTeX $\quad$ RIS \\
\hline
\end{tabular} 